\section{Estudos realizados em Portugal no âmbito do cateterismo venoso periférico: protocolo de scoping review}

Studies carried out in Portugal in the area of peripheral venous catheterization: scoping review protocol

Estudios realizados en Portugal en el campo de cateterización venosa periférica: protocolo de scoping review

Paulo Jorge dos Santos-Costa ${ }^{1,3}$

(D) https://orcid.org/0000-0003-0761-6548

Liliana Baptista Sousa ${ }^{1}$

(D) https://orcid.org/0000-0002-8914-6975

Inês Alexandra Figueira Marques ${ }^{2}$

(D) https://orcid.org/0000-0001-5690-3020

Anabela de Sousa Salgueiro-Oliveira ${ }^{1}$

(D) https://orcid.org/0000-0002-8231-8279

Pedro Miguel Dinis Parreira ${ }^{1}$

(D) https://orcid.org/0000-0002-3880-6590

Margarida Maria da Silva Vieira ${ }^{3}$

(D) https://orcid.org/0000-0002-9439-2804

Joăo Manuel Garcia Nascimento Graveto ${ }^{1}$

(D) https://orcid.org/0000-0001-6974-9421

${ }^{1}$ Unidade de Investigação em Ciências da Saúde: Enfermagem (UICISA: E), Escola Superior de Enfermagem de Coimbra (ESEnfC), Coimbra, Portugal

${ }^{2}$ Instituto de Pesquisa Clínica e Biomédica de Coimbra, Faculdade de Medicina da Universidade de Coimbra, Coimbra, Portugal

${ }^{3}$ Universidade Católica Portuguesa, Instituto de Ciências da Saúde, Porto, Portugal

\section{Autor de correspondência:}

Paulo Jorge dos Santos-Costa

E-mail: paulocosta@esenfc.pt

Recebido: 12.07 .19

Aceite: 04.03 .20

\section{Resumo}

Contexto: Apesar da sua natureza ubíqua, a prática de cateterizaçáo venosa periférica não é homogénea entre contextos clínicos internacionais. Em Portugal, a informaçáo referente à prática de cateterismo venoso periférico encontra-se dispersa na literatura, impossibilitando uma análise suficientemente compreensiva e abrangente da sua natureza e implicaçóes.

Objetivos: Mapear os estudos realizados em Portugal no âmbito do cateterismo venoso periférico.

Método de revisáo: Metodologia de scoping review proposta pelo Joanna Briggs Institute. Foi definido um protocolo adequado a cada base/repositório, que visa a identificação de estudos que respondam aos critérios delineados. O processo de análise da relevância, extraçáo e síntese dos dados será desenvolvido por revisores independentes. neste âmbito contribuirá para a identificaçáo dos principais indicadores de estrutura, processo e resultado descritos em estudos nacionais.

Conclusáo: Espera-se que esta revisão sustente o desenvolvimento de intervenções e revisões sistemáticas futuras que potenciem a eficácia/segurança dos cuidados prestados ao doente com cateter periférico.

Palavras-chave: cateterismo venoso periférico; revisão

\section{Abstract}

Background: Despite its ubiquitous nature, the practice of peripheral venous catheterization is not homogeneous among international clinical contexts. In Portugal, the information regarding the practice of peripheral venous catheterization is scattered in the literature, hindering efforts of a comprehensive analysis of its nature and implications.

Objetive: To map the studies developed in Portugal in the field of peripheral venous catheterization. Review method: Scoping review methodology proposed by the Joanna Briggs Institute. An adequate protocol was established for each base/repository in order to identify studies that meet the criteria outlined. The analysis of data relevance, extraction and synthesis will be performed by independent reviewers.

Presentation and interpretation of results: the mapping of the studies carried out in Portugal in this area will contribute to the identification of the main indicators of structure, process and outcome described in national studies.

Conclusion: It is expected that this review will support the development of future interventions and systematic reviews that enhance the efficacy/safety of the care provided to patients with peripheral catheter.

Keywords: catheterization, peripheral; review

\section{Resumen}

Contexto: Apesar de su naturaleza ubicua, la práctica del cateterismo venoso periférico no es homogénea en contextos clínicos internacionales. En Portugal, la información sobre la práctica del cateterismo venoso periférico está dispersa en la literatura, haciéndolo imposible un análisis lo suficientemente completo de su naturaleza e implicaciones.

Objetivos: Mapear los estudios realizados en Portugal en el ámbito del cateterismo venoso periférico. Método de revisión: Metodología de scoping review propuesta por Joanna Briggs Institute. Se definió un protocolo adecuado a cada base/repositorio para identificar estudios que respondan a los criterios delineados. El proceso de análisis da relevancia, extracción y síntesis de los datos será desarrollado por revisores independientes.

Presentación e interpretación de los resultados: El mapeo de los estudios realizados en Portugal en esta área contribuirá a la identificación de los principales indicadores de estructura, proceso y resultado descritos en estudios nacionales.

Conclusión: Se espera que esta revisión contribuya al desarrollo de futuras intervenciones y revisiones sistemáticas que mejoren la eficacia/seguridad de la atención del paciente con catéter periférico.

Palabras clave: cateterismo periférico; revisión
Apresentaçáo e interpretaçáo dos resultados: $\mathrm{O}$ mapeamento dos estudos realizados em Portugal

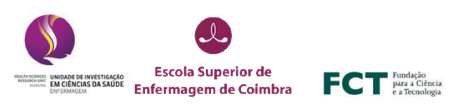

Como citar este artigo: Santos-Costa, P., Sousa, L. B., Marques, I. A., Salgueiro-Oliveira, A., Parreira, P., Vieira, M., \& Graveto, J. (2020). Estudos realizados em Portugal no âmbito do cateterismo venoso periférico: protocolo de scoping review. Revista de Enfermagem Referência, 5(3), e20004. doi:10.12707/RV20004 


\section{Introduçáo}

Desde a sua conceção inicial, em 1658, que o cateter venoso periférico (CVP) se afigura como uma das descobertas mais revolucionárias na história da medicina contemporânea, sendo utilizado recorrentemente na administração de fármacos, fluídos ou hemoderivados, assim como na colheita de sangue (Rivera, Strauss, Zundert, \& Mortier, 2005).

Anualmente, são inseridos cerca de 1.5 biliōes de CVPs em todo o mundo, ainda que estes números estejam sub-reportados ao nível Europeu (Alexandrou et al., 2015), estimando-se que $60 \%$ a $90 \%$ dos doentes internados necessitem de, pelo menos, um CVP para cumprir o seu plano terapêutico (Helm, Klausner, Klemperer, Flint, \& Huang, 2015) sendo uma prática clínica abrangente e global. Todavia, os CVPs não estão isentos de riscos, existindo evidência de complicaçóes recorrentes como flebite, obstrução, extravasamento ou infeção nosocomial associada à corrente sanguínea (INCS). Estas complicaçóes apresentam um impacte significativo nas taxas de morbilidade e mortalidade dos doentes, com evidentes repercussóes socioeconómicas (Helm et al., 2015). Acresce referir que apesar das complicaçóes associadas à presença dos CVPs, estima-se ainda que até $90 \%$ dos CVPs são removidos prematuramente, antes de se cumprir o plano terapêutico que motivou a sua inserçấo (Alexandrou et al., 2018).

Face a esta realidade, Jones (2018) estabelece que, além das características sociodemográficas e condição clínica dos doentes, a qualidade e eficácia do cateterismo venoso periférico são condicionadas pelas práticas e dispositivos médicos utilizados pelos profissionais de saúde durante a inserção, manutenção e remoção do CVP. Assente nesta perspetiva, os resultados encontrados na literatura deverão ser analisados com ressalvas, dado que a gestáo do cateterismo venoso periférico não é uniforme entre contextos internacionais (Alexandrou et al., 2015). A título de exemplo, ainda que um número considerável de publicaçóes neste âmbito temático seja realizado nas regiōes da Austrália e Nova Zelândia, Alexandrou e colaboradores (2018) identificaram que apenas 26\% dos CVPs eram inseridos por enfermeiros. De igual modo, no Reino Unido, o cateterismo venoso periférico pode ser realizado por enfermeiros, médicos, assistentes operacionais e técnicos de radiologia (Cooper, Whitfield, Newton, Chiarella, \& Machaczek, 2016). A heterogeneidade reportada a este nível mantem-se em contextos clínicos de países europeus, norte-americanos, africanos e asiáticos (Alexandrou et al., 2018).

Em Portugal, a cateterização venosa periférica constitui uma intervenção interdependente da prática de enfermagem $(\mathrm{Or}-$ dem dos Enfermeiros, Mesa do Colégio da Especialidade em Enfermagem de Saúde Infantil e Pediátrica, 2017). Cabe aos enfermeiros a prestação de cuidados à pessoa com necessidade de cateterização venosa periférica, desde a inserção do cateter até à sua remoção, devendo ser estes os profissionais a monitorizar possíveis alteraçōes decorrentes (Ordem dos Enfermeiros, Mesa do Colégio da Especialidade em Enfermagem de Saúde Infantil e Pediátrica, 2017). Para este efeito, é-lhes exigida atualizaçâo contínua sobre esta temática, perspetivando a procura permanente da excelência no seu exercício profissional (Ordem dos Enfermeiros, Mesa do
Colégio da Especialidade em Enfermagem de Saúde Infantil e Pediátrica, 2017). Todavia, identificar resultados sensíveis aos cuidados de enfermagem nem sempre é um processo linear (Irvine, Sidani, \& Hall, 1998). Sustentadas no modelo de qualidade proposto por Donabedian, em 1980, as autoras afirmam que a análise dos resultados sensíveis aos cuidados de enfermagem deverá atender a componentes de estrutura (variáveis associadas aos enfermeiros, às pessoas doentes e à organização de saúde), de processo (papel independente, dependente ou interdependente dos enfermeiros), e de resultados, sensíveis aos cuidados de enfermagem, entre os quais destacam a prevenção de complicaçóes. De acordo com Irvine e colaboradoras (1998), ainda que os enfermeiros não sejam os únicos responsáveis pela ocorrência de complicaçóes, são estes os profissionais melhor posicionados para garantir a supervisão contínua dos cuidados de saúde prestados.

Todavia, a evidência científica produzida em Portugal no âmbito do cateterismo venoso periférico encontra-se dispersa na literatura, impossibilitando a realização de uma avaliação abrangente e fundamentada da influência de variáveis de estrutura e processo nos outcomes clínicos (resultados) associados ao cateterismo venoso periférico. $\mathrm{Na}$ sequência de uma pesquisa preliminar na JBI Database of Systematic Reviews and Implementations Reports, Cochrane Database of Systematic Reviews, na CINAHL (via EBSCO) e na MEDLINE (via PubMED), não foram encontradas quaisquer tipos de revisões da literatura (publicadas ou em realização/serem realizadas) neste âmbito temático.

De acordo com Apóstolo (2017), aquando da existência de lacunas na avaliação de uma área ou foco, o mapeamento sistematizado da evidência científica produzida poderá constituir um ponto de partida para a análise da extensão, alcance e natureza da atividade de investigação realizada até à data. Desta forma, pretende-se realizar uma scoping review, orientada pela metodologia proposta pelo Joanna Briggs Institute (Peters et al., 2017), com o objetivo de mapear os estudos desenvolvidos em Portugal no âmbito do cateterismo venoso periférico, analisando e sistematizando os principais indicadores de estrutura, processo e resultado identificados.

Mais especificamente, esta revisão pretende dar resposta às seguintes questôes: i) Quais são as principais áreas de foco dos estudos realizados em Portugal no âmbito do cateterismo venoso periférico?; ii) Em Portugal, quais os contextos clínicos onde são realizados estudos relativos ao cateterismo venoso periférico?; iii) Que práticas profissionais são adotadas ao nível da inserção, manutenção e remoção destes dispositivos em Portugal?; iv) Quais sấo os dispositivos médicos utilizados em Portugal no âmbito do cateterismo venoso periférico?; e v) Quais são os outcomes clínicos reportados em estudos desenvolvidos em Portugal no âmbito do cateterismo venoso periférico?

\section{Método de revisão sistemática}

Em 2005, Arksey e O'Malley desenvolveram o primeiro enquadramento metodológico para scoping reviews, delineando quatro razóes principais para a sua realizaçáo: i) analisar a amplitude, alcance e natureza da produção 
científica publicada num determinado tema; ii) avaliar a relevância, viabilidade e potenciais custos da realização de uma revisão sistemática da literatura; iii) sumariar e disseminar a produção científica existente num âmbito temático, evidenciando os seus principais resultados junto de decisores políticos, profissionais e principais consumidores, os quais podem não despender do tempo e recursos para investir na sua procura; e iv) identificar lacunas na literatura, contribuindo para a definição de próximas etapas e estudos a desenvolver numa determinada temática. Com igual relevância, as scoping reviews podem ser utilizadas para analisar o modo como a investigaçáo tem sido desenvolvida num determinado tema ou área científica (Munn et al., 2018). Neste desiderato, também na área científica de enfermagem, as scoping reviews afiguram-se como uma etapa importante em qualquer projecto de investigaçáo, fundamentando o desenvolvimento de estudos futuros que procurem contribuir para a investigação, formação, prática e decisão política num determinado segmento temático (Peterson, Pearce, Ferguson, \& Langford, 2017).

\section{Estratégia de pesquisa e identificaçáo dos estudos} Seguindo a metodologia proposta pelo Joanna Briggs Institute (Peters et al., 2017), serão definidos os participantes, o conceito e o contexto da revisão (mnemónica PCC). Nesta lógica, no que respeita aos participantes, serão incluídos todos os estudos que incluam profissionais de saúde com competências para realizar a inserção, manutenção e remoção de CVPs. Relativamente ao conceito, pretende-se a inclusão de estudos realizados no âmbito do cateterismo venoso periférico de pessoas, que reportem indicadores de estrutura, processo ou resultado associados. Relativamente ao contexto, serão integrados estudos realizados em qualquer contexto clínico e área geográfica do território português.

Respeitante ao tipo de estudo, esta revisão irá considerar estudos de natureza primária, como estudos observacionais, estudos descritivos transversais ou longitudinais, estudos quasi-experimentais antes/após, estudos de caso e estudos randomizados controlados. Além disto, serão incluídas para análise revisóes da literatura que integrem estudos primários desenvolvidos neste âmbito temático em Portugal.

No que respeita à estratégia de pesquisa e identificação dos estudos, serão utilizadas as bases de dados eletrónicas CINAHL e MedicLatina (via EBSCO), MEDLINE (via PubMed), SciELO, Scopus, LILACS e Cochrane Central Register of Controlled Trials. Por sua vez, de modo a mapear literatura náo-publicada como teses e dissertaçóes académicas desenvolvidas em Portugal, será replicada a estratégia de pesquisa no Repositório Científico de Acesso Aberto de Portugal, Banco de Teses da CAPES e OpenGrey. De modo a potenciar uma análise compreensiva do fenómeno em análise não serão considerados limites temporais para a pesquisa e seleçáo de artigos. No que concerne à língua de publicação, serão incluídos artigos escritos em português, inglês, espanhol ou francês.

A pesquisa será desenvolvida em três etapas, a saber: i) pesquisa limitada nas bases de dados MEDLINE (via PubMed) e CINAHL (via EBSCO), de modo a identificar as palavras e termos de indexação mais frequentemente utilizados (Tabela 1); ii) as palavras e termos de indexação identificados serão conjugados numa estratégia de pesquisa única, de acordo com as especificidades de cada basel repositório selecionado; iii) a lista de referências de cada estudo selecionado será analisada por dois revisores independentes de modo a incluir potenciais estudos adicionais.

\section{Tabela 1}

Exemplo de pesquisa inicial na MEDLINE (via PubMed) a 17 de dezembro de 2019

\begin{tabular}{|c|c|}
\hline Query & Results \\
\hline $\begin{array}{l}\text { Search: }((((\text { Catheters, Indwelling[MeSH Major Topic]) OR (Catheters[MeSH Major Topic])) OR (Catheter-Relat- } \\
\text { ed Infections[MeSH Major Topic])) OR (Catheter Obstruction[MeSH Major Topic])) OR (Vascular Access Devic- } \\
\text { es[MeSH Major Topic])) OR (Catheterization, Peripheral[MeSH Major Topic])) OR ("peripheral venous catheter"[Ti- } \\
\text { tle/Abstract]) OR ("venous line"[Title/Abstract])) OR (“venous access"[Title/Abstract])) OR ("venous device”[Title/ } \\
\text { Abstract])) OR ("peripheral catheter"[Title/Abstract])) OR (cannula*[Title/Abstract])) OR (PIVC[Title/Abstract])) } \\
\text { OR (PVC[Title/Abstract])) OR (canula*[Title/Abstract])) OR ("peripheral intravenous catheter"[Title/Abstract])) OR } \\
\text { ("venous catheter"[Title/Abstract])) OR ("intravenous catheter"[Title/Abstract])) AND Portugal }\end{array}$ & 284 \\
\hline
\end{tabular}

\section{Seleçáo de estudos}

Após a pesquisa nas bases de dados e repositórios, os estudos identificados serão introduzidos no software Mendeley (Elsevier, Holanda), através do qual serão eliminados os artigos duplicados. De seguida, dois revisores independentes irão analisar os títulos e resumos dos estudos encontrados, confrontando-os com os critérios de inclusão previstos para a revisão. Todos os estudos que atendam aos critérios definidos serão analisados por texto completo. Os estudos de texto completo que não atendam aos critérios de inclusão serão excluídos e as razões serão identificadas. Os resultados deste processo serão apresentados com recurso à Preferred Reporting Items for Systematic Reviews and
Meta-Analyses extension for Scoping Reviews (PRISMA-ScR) checklist (Tricco et al., 2018). Quaisquer discordâncias que surjam entre os revisores serão resolvidas por meio de discussão ou com um terceiro revisor.

\section{Extraçáo dos dados}

A extração de dados será realizada por dois revisores independentes, com recurso a um instrumento construído pelos investigadores visando dar resposta ao objetivo e questóes de revisão delineadas (Figura 1). Durante esta fase, o instrumento poderá sofrer alteraçóes de acordo com eventuais necessidades emergentes sentidas pelos investigadores. 


\section{Título da Revisão}

Estudos realizados em Portugal no âmbito do cateterismo venoso periférico: protocolo de scoping review

\section{Questōes de Revisão:}

i) Quais são as principais áreas de foco dos estudos realizados em Portugal no âmbito do cateterismo venoso periférico?

ii) Em Portugal, quais os contextos clínicos onde são realizados estudos relativos ao cateterismo venoso periférico?

iii) Que práticas profissionais são adotadas ao nível da inserção, manutenção e remoção destes dispositivos em Portugal?

iv) Quais são os dispositivos médicos utilizados em Portugal no âmbito do cateterismo venoso periférico?

v) Quais são os outcomes clínicos reportados em estudos desenvolvidos em Portugal no âmbito do cateterismo venoso periférico?

\section{Critérios de inclusão:}

- Participantes

Estudos que incluam profissionais de saúde com competências para realizarem a inserção, manutenção e remoção de CVPs.

- Conceito

Estudos realizados no âmbito do cateterismo venoso periférico de pessoas, que reportem indicadores de estrutura, processo ou resultado associados.

- Contexto

Estudos realizados em qualquer contexto clínico e área geográfica do território Português.

\section{Extracão de detalhes e características do estudo}

\begin{tabular}{|l|l|}
\hline Autores & \\
\hline Ano de Publicação & \\
\hline Tipo de Estudo & \\
\hline Objetivos do estudo & \\
\hline Número de participantes & \\
\hline $\begin{array}{l}\text { Conceitos relevantes para a questão de } \\
\text { revisão }\end{array}$ & \\
\hline
\end{tabular}

Figura 1. Instrumento criado pelos investigadores para a extração de dados.

De forma transversal, os dados extraídos irão incidir sobre as características da população em estudo, desenho e resultados relevantes para dar resposta à questão e objetivos específicos traçados. Durante o processo de extração (quando e sempre que necessário) os autores dos estudos selecionados serão contactados para clarificar dados ou disponibilizar informaçóes adicionais. As divergências que possam emergir nesta fase serão resolvidas através de diálogo entre revisores ou, caso não haja consenso, recorrendo a um terceiro elemento.

\section{Síntese dos dados}

Os dados mapeados seráo apresentados de forma narrativa, alinhada com o objetivo e foco da scoping review, com recurso a tabelas e/ou gráficos. Semelhante à fase anterior, a síntese e apresentação dos dados será realizada através de consenso entre dois revisores. Quaisquer divergências serão resolvidas com recurso a um terceiro elemento.

Para as questóes "Em Portugal, quais os contextos clínicos onde são realizados estudos relativos ao cateterismo venoso 
periférico?" e "Quais são as principais áreas de foco dos estudos realizados em Portugal no âmbito do cateterismo venoso periférico?”, as tabelas e gráficos poderão incluir dados indicados na Tabela 2.

Tabela 2

Grelha de sintese de dados para primeira e segunda questäo de revisäo

Focos identificados

Estudo

Contexto Clínico

(por favor, descreva o foco identificado)

Estrutura Processo Resultado

Para a questão de revisão "Que práticas profissionais são

destes dispositivos em Portugal?”, as tabelas e gráficos adotadas ao nível da inserção, manutenção e remoção poderão incluir dados indicados na Tabela 3.

Tabela 3

Grelha de sintese de dados para a terceira questão de revisão

\begin{tabular}{lcccc}
\hline \multirow{2}{*}{ Estudo } & $\begin{array}{c}\text { Profissional de saúde responsável } \\
\text { pela gestáo do CVP }\end{array}$ & \multicolumn{2}{c}{$\begin{array}{c}\text { Práticas profissionais identificadas } \\
\text { (por favor, descreva as práticas identificadas) }\end{array}$} \\
\cline { 2 - 4 } & Inserção de CVP & Manutenção de CVP & Remoçáo do CVP \\
\hline
\end{tabular}

Para as questões de revisão "Quais são os dispositivos médicos utilizados em Portugal no âmbito do cateterismo venoso periférico?" e "Quais são os outcomes clínicos reportados em estudos desenvolvidos em Portugal no âmbito do cateterismo venoso periférico?", as tabelas e gráficos poderăo incluir dados indicados na Tabela 4.

Tabela 4

Grelha de sintese de dados para a quarta e quinta questão de revisão

\begin{tabular}{|c|c|c|c|c|c|c|}
\hline \multirow[b]{2}{*}{ Estudo } & \multirow[b]{2}{*}{$\begin{array}{l}\text { Dispositivos médicos } \\
\text { identificados }\end{array}$} & \multicolumn{5}{|c|}{ Outcomes clínicos reportados } \\
\hline & & Flebite & $\begin{array}{c}\text { Infeçấo nosocomial } \\
\text { da Corrente Sanguínea }\end{array}$ & Extravasamento & Infiltração & $(\ldots)$ \\
\hline
\end{tabular}

\section{Apresentaçáo e interpretaçáo dos resultados}

O mapeamento da produçấo científica desenvolvida em Portugal no âmbito do cateterismo venoso periférico, contribuirá para a análise e discussão das práticas profissionais vigentes, características dos dispositivos utilizados e das principais complicações identificadas em contexto nacional.

\section{Conclusão}

Espera-se que esta scoping review constitua um ponto de partida profícuo para a análise e sistematização dos principais indicadores de estrutura, processo e resultado identificados em estudos nacionais no âmbito do cateterismo venoso periférico. Para além do contributo para a área da investigação, espera-se que esta investigação contribua para a melhoria da prática clínica ligada à presença do $\mathrm{CPV}$, permitindo a identificação dos principais desafios e lacunas que poderão justificar a necessidade de se desenvolverem programas de formação profissional adequados aos contextos clínicos e organizacionais contemplando os recursos presentes. Com igual relevância, os resultados obtidos com esta revisão poderão substanciar a necessidade de se desenvolver, emitir e adoptar uma norma nacional que uniformize os cuidados ao doente portador de CVP (até à data inexistente), potenciando cuidados de maior eficácia, segurança, eficiência e qualidade para os doentes e para os profissionais de saúde.

\section{Contribuiçáo de autores}

Conceptualização: Santos-Costa, P.

Tratamento de dados: Santos-Costa, P., Sousa, L. B., Marques, I. A.

Metodologia: Santos-Costa, P., Vieira, M., Graveto, J.

Redação - preparação do rascunho original: Santos-Costa, P. Redação - revisão e edição: Santos-Costa, P., Sousa, L. B., Marques, I. A., Salgueiro-Oliveira, A., Parreira, P., 
Vieira, M., Graveto, J.

Supervisão: Vieira, M., Graveto, J.

\section{Agradecimentos}

Este protocolo foi realizado no âmbito do percurso doutoral do primeiro autor (bolsa SFRH/BD/136487/2018), financiado pela Fundação para a Ciência e a Tecnologia, IP (FCT), através de fundos dos programas POPH/FSE. Os autores gostariam de agradecer o apoio do eixo de desenvolvimento estratégico para a síntese da evidência (PCEBP/JBI) da Unidade de Investigação em Ciências da Saúde: Enfermagem (UICISA: E), acolhida pela Escola Superior de Enfermagem de Coimbra (ESEnfC).

\section{Referências bibliográficas}

Alexandrou, E., Ray-Barruel, G., Carr, P., Frost, S., Inwood, S., Higgins, N., ... Rickard, C. M. (2015). International prevalence of the use of peripheral intravenous catheters. Journal of Hospital Medicine, 10(8), 530-533. doi: 10.1002/jhm.2389

Alexandrou, E., Ray-Barruel, G., Carr, P., Frost, S., Inwood, S., Higgins, N., ... OMG Study Group (2018). Use of short peripheral intravenous catheters: Characteristics, management, and outcomes worldwide. Journal of Hospital Medicine, 13(5). doi: 10.12788/jhm.3039

Apóstolo, J. (2017). Sintese da evidência no contexto da translaçâo da ciência. Coimbra, Portugal: Escola Superior de Enfermagem de Coimbra.

Arksey, H., \& O’Malley, L. (2005). Scoping studies: Towards a methodological framework. International Journal of Social Research Methodology, 8(1), 19-32. doi: 10.1080/1364557032000119616

Cooper, D., Whitfield, M., Newton, D., Chiarella, J., \& Machaczek, K. (2016). Introduction of a non-ported peripheral intravenous catheter with multi-use blood control septum offers improvements in the overall efficiency of the procedure and is clinically well accepted. International Journal Of Healthcare Technology And Management, 15(3), 177. doi: 10.1504/ijhtm.2016.078336
Helm, R., Klausner, J., Klemperer, J., Flint, L., \& Huang, E. (2015). Accepted but unacceptable. Journal of Infusion Nursing, 38(3), 189-203. doi: 10.1097/nan.0000000000000100

Irvine, D., Sidani, S., \& Hall, L. (1998). Linking outcomes to nurses' roles in health care. Nursing Economics, 16(2), 58-64.

Jones, R. (2018). Short peripheral catheter quality and economics. Journal Of Infusion Nursing, 41(6), 365-371. doi: 10.1097/ nan.0000000000000303

Munn, Z., Peters, M., Stern, C., Tufanaru, C., McArthur, A., \& Aromataris, E. (2018). Systematic review or scoping review? Guidance for authors when choosing between a systematic or scoping review approach. BMC Medical Research Methodology, 18(1). doi: 10.1186/s12874-018-0611-x

Ordem dos Enfermeiros, Mesa do Colégio da Especialidade em Enfermagem de Saúde Infantil e Pediátrica. (2017). Parecer $n^{\circ}$ 6/ 2017: Aplicação de calor local em seroma provocado por infiltração de soroterapia em lactentes. Recuperado de https://www.ordemenfermeiros.pt/media/4806/parecer-n\%C2\%BA-6_2017.pdf

Peters, M., Godfrey, C., McInerney, P., Baldini Soares, C., Khalil, H., \& Parker, D. (2017). Scoping reviews. In E. Aromataris \& Z. Munn (Eds.), Joanna Briggs Institute Reviewer's Manual. Recuperado de https://reviewersmanual.joannabriggs.org/

Peterson, J., Pearce, P., Ferguson, L., \& Langford, C. (2017). Understanding scoping reviews. Journal of The American Association Of Nurse Practitioners, 29(1), 12-16. doi: 10.1002/2327-6924.12380

Rivera, A., Strauss, K., Zundert, A., \& Mortier, E. (2005). The history of peripheral intravenous catheters: How little plastic tubes revolutionized medicine. Acta Anaesthesiologica Belgica., 56(3), 271-282.

Tricco, A., Lillie, E., Zarin, W., O’Brien, K., Colquhoun, H., Levac, D., \& Straus, S. (2018). PRISMA Extension for Scoping Reviews (PRISMA-ScR): Checklist and Explanation. Annals Of Internal Medicine, 169(7), 467. doi: 10.7326/m18-0850 